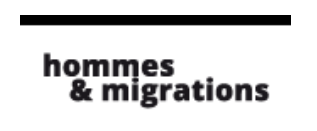

\section{Hommes \& migrations}

Revue française de référence sur les dynamiques

migratoires

$1324 \mid 2019$

Religion et discrimination

\title{
Yves Tsao, Les travailleurs chinois recrutés par la France pendant la Grande Guerre
}

Aix-en-Provence, Presses Universitaires de Provence, 2018, 366 p., $25 €$

\section{Yasmine Achouche}

\section{(2) OpenEdition}

Journals

Édition électronique

URL : https://journals.openedition.org/hommesmigrations/8752

DOI : $10.4000 /$ hommesmigrations.8752

ISSN : 2262-3353

Éditeur

Musée national de l'histoire de l'immigration

Édition imprimée

Date de publication : 1 janvier 2019

Pagination : 227

ISBN : 978-2-919040-44-5

ISSN : 1142-852X

Référence électronique

Yasmine Achouche, «Yves Tsao, Les travailleurs chinois recrutés par la France pendant la Grande Guerre », Hommes \& migrations [En ligne], 1324 | 2019, mis en ligne le 01 janvier 2019, consulté le 08 janvier 2022. URL : http://journals.openedition.org/hommesmigrations/8752 ; DOI : https://doi.org/ 10.4000/hommesmigrations.8752 


\section{Livres}

\section{J'apprends le français}

Marie-France Etchegoin, Paris, JC Lattès, 2018, 200 pages, 17 euros.

Benoit Cohen Mohammad, ma mère et moi

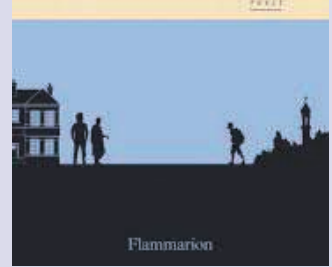

«Parfois je hais le français. Que de chausse-trappes, d'afféteries, de chichiteries. Toutes ces lettres silencieuses ou capricieuses.» Au-delà du livre documentaire qu'elle nous propose sur l'accueil des migrants en France en 2018, la journaliste Marie-France Etchegoin donne dans ce livre une belle leçon d'humilité pour tous ceux qui font œuvre d'hospitalité. Comme d'autres habitants du 20 e arrondissement de Paris, elle décide de participer à l'aventure du Centre Jean Quarré, géré par Emmaüs solidarité, qui s'installe dans un ancien lycée hôtelier de la place des Fêtes en 2016 afin d'accueillir une centaine de migrants en hébergement d'urgence (voir notre article dans $H \& M$, $n^{\circ}$ 1323).

Elle n'a jamais enseigné le français mais décide de donner bénévolement un cours hebdomadaire à un groupe d'une dizaine de personnes pendant delongs mois. Pour se souvenir de l'identité des élèves qui viennent plus ou moins régulièrement à son cours, elle leur donne des surnoms. Il y a Le Doux, Superman, La Flèche, Le Polyglotte, Le Musicien, etc. Elle apprend par bribes l'histoire de ces hommes qui ont traversé la guerre, les périls d'un voyage interdit et les affres d'un accueil plus que tiède enEurope. Elle glane des mots au détour d'une conversation en tête-à-tête avant ou après le cours, voire pendant.

Elle n'applique pas toujours ce que lui recommande l'équipe qui gère les cours de français, consciente que ces moments qu'elle partage avec ces hommes exilés, dans l'attente de papiers, d'un travail et tout simplement d'un avenir, dépassent le simple cours de grammaire ou la transmission de nouveaux mots. «Parler n'est jamais innocent, écrit Marie-France Etchegoin dans le chapitre intitulé «Les Mots». Apprendre à parler une nouvelle langue encore moins.» L'auteur cite Hannah Arendt, la philosophe juive allemande exilée aux États-Unis pendant la Seconde Guerre mondiale, qui décrit le réfugié comme un être qui a perdu «sa langue maternelle, c'est-à-dire [ses] réactions naturelles, la simplicité des gestes, l'expression spontanée de [ses] sentiments».

Il faut réapprendre à dire, l'indicible parfois, dans une langue qui n'est pas la sienne. L'auteur se souvient avec émotion du jour où Ibrahim le Soudanais s'obstine à dire: "je n'ai PAS de femme, je n'ai PAS d'enfant» alors qu'il devrait dire «je n'ai PLUS de femme, je n'ai PLUS d'enfants». Il a très bien compris la différence entre les deux mots, mais il choisit de parler de la mort insoutenable de ceux qu'il aimait avec la négation pas.

Pour survivre.

Dans ce livre, l'auteur annonce tout de suite qu'elle ne veut pas seulement raconter la souffrance mais aussi la dignité et le courage des hommes qu'elle rencontre. Elle doute parfois de la légitimité de sa présence et se reproche souvent ses maladresses. Elle s'en veut surtout pour la bonne conscience que lui procure cette action. Elle fait parler son «inquisiteur» intérieur: «Les migrants n'ont pas besoin de charité, ils ont besoin de droits.
L'altruisme étouffe la structuration des luttes. L'altruisme est paternaliste, néocolonialiste. Toi, et tous les autres bénévoles pleins de bons sentiments, toi et toutes ces associations qui acceptent de gérer les quelques centres donnés en aumône aux migrants, vous êtes des rustines sur l'inacceptable.» Marie-France Etchegoin fait plus qu'être une rustine, elle rencontre des hommes qu'elle n'aurait jamais rencontrés et nous raconte qu'ils ont changé sa vie. Elle nous donne aussi plein d'informations et de noms d'associations qui œuvrent pour fournir des outils aux migrants et les sortir de leur condition de «victimes» pour devenir «acteurs» de la société dans lesquels ils ont décidé de vivre. Ce livre contribue à témoigner et à informer le mouvement citoyen qui se développe, notamment à Paris, depuis la crise de l'accueil des migrants en 2015.

Catherine Guilyardi

\section{Contre la haine. Plaidoyer pour l'impur}

Carolin Emcke, Paris, Seuil, 2017, 224 pages, 17 euros.

\section{contre la haine}

plaidoyer pour l'impur \begin{tabular}{l} 
Carolin \\
Emcke \\
\hline
\end{tabular}

ouvrage, qui a suscité un grand intérêt en Allemagne à sa parution, s'inscrit dans le contexte allemand de montée des extrémismes politiques et du racisme contre les immigrés qui n'est pas forcément compris dans sa complexité en France. D'où la traduction et la 
publication salutaires de cet essai. Il interroge le paradoxe entre la politique d'ouverture des frontières et d'accueil des migrants menée par la chancelière Angela Merkel en 2015 (et aujourd'hui stoppée) et la montée, dans l'opinion allemande, d'une haine tenace comme objet dérivatif d'un malaise plus profond fondé sur le déclassement social et la peur de l'impur. Pour répondre à cette question, Carolin Emcke, journaliste mais aussi philosophe, politologue et écrivaine (elle a étudié avec Jürgen Habermas), mobilise utilement les travaux de recherche sur le racisme et les discriminations en Allemagne et en Europe. L'apport de philosophes français (comme Jacques Derrida cité en exergue) et allemands enrichit son analyse sur la haine des autres. La littérature contemporaine aurait pu également être sollicitée comme espace defabrication d'un imaginaire sur les sociétés cosmopolites.

Cet ouvrage rappelle qu'il faut étudier ces phénomènes de rejet dans leur globalité: examiner les sources et les modalités différentes qui construisent et alimentent lahaine et qui en permettent la diffusion («les pourvoyeurs mais aussi les profiteurs de haine»); étudier les évolutions des opinions (idéologies, idées reçues et mystifications) et leurs conséquences pratiques, quotidiennes, dans la vie des populations («l'enfermement, des petites techniques basses de l'exclusion par les gestes, les habitudes, les pratiques et les convictions... »). Enfin, analyser la réception de ces discours violents et attitudes racistes par les populations ciblées, les immigrés et leurs familles.

L'auteure constate, comme en France, une libération de la société allemande du «corset des conventions sociales» héritées de la Seconde Guerre mondiale («on hait désormais ouvertement et sans vergogne»). Elle explique comment le verrou des interdits moraux a pu sauter, sur la tolérance, le respect des autres, l'exigence de l'égalité des droits et des chances, et souligne la responsabilité des médias et des partis politiques. L'Allemagne serait victime d'une «tolérance ambiguë» portée par les partis de gauche sur le soutien des minorités et de la diversité culturelle et religieuse.

Elle fait une analyse très pertinente des mécanismes de (sur) visibilité

- invisibilité - qui jouent sur les représentations de l'immigration en Allemagne et alimentent la haine des étrangers. Ces mécanismes surfent sur les normes et les valeurs dominantes, sur les récits nationaux qui fondent et entretiennent la communauté nationale (le «nous»), sur les cadres d'interprétation qui accordent un rôle, une place et une reconnaissance ou non à certains groupes sociaux dans la société allemande. Elle met en lumière les mécanismes de déshumanisation des réfugiés devenus des individus invisibles, appréhendés uniquement comme des représentants (d'une culture, d'une religion pour ne pas dire d'une race) de populations marginalisées qui participent largement «au rétrécissement de la vision» et à un imaginaire de l'invasion.

Carolin Emcke étudie dans son essai la gradation dans les attitudes de rejet des étrangers. Elle introduit cette notion d'inquiétude comme «sentiment-écran», en reprenant aussi la notion de «dégoût projectif» empruntée à Martha Nussbaum. Comprendre la complexité des composantes de cette inquiétude permet de combattre plus efficacement le racisme, en distinguant ce qui relève de l'angoisse du déclassement social, de la privation de droits (ou de libertés pour les femmes par exemple vis-à-vis des musulmans), du mécontentement face aux inégalités sociales, du sentiment de marginalisation politique et de défiance vis-à-vis des représentations politiques. Le jeu du pur, de l'homogène et de l'authenticité est pour elle une composante du rapport à l'égalité sociale, et les questions sociales apparaissent comme le levier principal de ces mouvements de haine.

Elle propose une approche sociogéographique différenciée du racisme. Cela signifie que la radiographie des éléments structurels ou conjoncturels qui expliquent l'émergence du racisme varie selon les régions, les villes et les quartiers, et selon les groupes sociaux sur le territoire allemand. Les médias allemands contribuent à «nationaliser» et à amplifier ces situations si diverses sur le terrain. Il serait également intéressant de voir comment les réseaux sociaux et Internet constituent un accélérateur des opinions de rejet. Le chapitre sur l'arrivée du bus à Clausnitz est tout à fait exemplaire: en décortiquant cette scène du point de vue de tous les acteurs, y compris la police et ceux qui ne sont pas venus, Carolin Emcke nous permet de prendre conscience de l'épaisseur de «la haine à l'œuvre», des émotions, de la violence des attitudes et de leurs effets dévastateurs sur les réfugiés.

Cet essai permet de revisiter les actions de prévention et de lutte contre le racisme en montrant pourquoi il ne faut pas dévaloriser les populations inquiètes, celles qui sont passives et ne réagissent pas face à ces débordements de violence et de haine par des arguments moraux. Car le refus de s'engager est propre au mécanisme des foules. En revanche, elle dresse un tableau de la réalité des mouvements sociaux de revendication des droits et de soutien des immigrés en Allemagne qui ont du mal à mobiliser aujourd'hui. Les réflexions de Carolin Emcke peuvent les aider à formuler de nouvelles stratégies d'action collective et des discours publics adaptés pour lutter plus efficacement contre cette lame de fond.

\section{Marie Poinsot}




\section{La souffrance psychique des exilés : une urgence de santé publique}

Centre Primo Levi, Médecins du monde, Paris, Centre Primo

Levi/Médecins du monde, 2018, 19 pages.

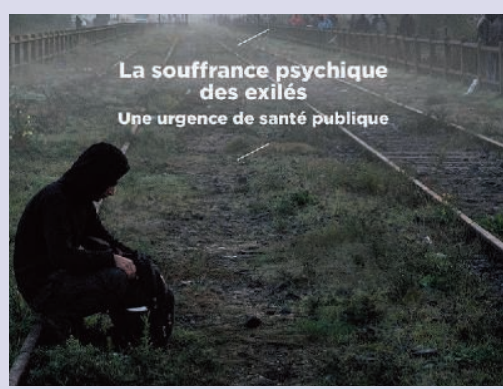

À l'occasion de la journée mondiale des réfugiés, Médecins du monde et le Centre Primo Levi, spécialisé dans le suivi des personnes victimes de torture, publient un rapport sur la souffrance psychique des exilés en France. Se nourrissant des expériences de terrain de leurs personnels respectifs, l'état des lieux présenté met en lumière l'urgence de cet enjeu de santé publique.

"Toutes les personnes que nous rencontrons ont été touchées dans leur intégrité physique et

psychique», décrit le Docteur Daniel Brehier, psychiatre à Médecins du monde. Après avoir fui des événements insoutenables dans leur pays d'origine, ils ont vécu des trajectoires de migrations au moins tout aussi violentes, "passant souvent par la Libye ou le Maroc, où les violences physiques, la torture et les viols semblent massifs» (p. 6). Et les souffrances se poursuivent, voire s'aggravent, une fois arrivés en France, où la très grande précarité sociale et administrative dans laquelle vivent les réfugiés les prive d'une stabilité indispensable pour se reconstruire.

Les syndromes post-traumatiques qui découlent de l'expérience de l'exil prennent des formes multiples: états anxieux, troubles somatiques, réminiscences des scènes vécues, auxquels s'ajoute le plus souvent une grande difficulté à retrouver la cohérence de son identité, après un parcours de vie décousu et chaotique. La difficulté que rencontrent les exilés pour la mise en mots de leurs expériences, rendue problématique pour les réfugiés, est d'ailleurs pointée à de nombreuses reprises par les médecins ayant participé au rapport. Ceux-ci dénoncent les souffrances supplémentaires induites par la procédure d'évaluation de la demande d'asile, qui repose sur la mise en récit de ce passé douloureux, et qui, lorsqu'elle se solde par un refus administratif, s'apparente à une véritable négation du trauma qui a été vécu.

La situation des mineurs non accompagnés (MNA) est à ce titre particulièrement préoccupante, et leur prise en charge est d'autant plus urgente que ces violences surviennent à un moment clé de leur développement psychique. Cependant, le Docteur Omar Guerrerro souligne également la très grande plasticité de ces jeunes, qui font preuve d'une forte résilience s'ils sont suivis de manière adaptée. Le psychiatre Daniel Brehier insiste ainsi sur la nécessité de bien préparer en amont ceux qui accompagnent les exilés au quotidien (que ce soit sur le plan psychologique ou pratique), afin d'éviter les «effets radioactifs» (p. 15) qui peuvent affecter tant les proches que les professionnels confrontés à des récits d'expériences insoutenables.

Le rapport pointe donc directement les nombreuses défaillances du système de prise en charge psychologique actuel: repérage et orientation insuffisants des personnes en souffrance, caractère chaotique des parcours de soins, faible recours à l'interprétariat professionnel, accès difficile à une couverture maladie pour les étrangers... Ses auteurs regrettent surtout que la «[santé] des personnes exilées demeure trop souvent un enjeu de polémique politicienne avant d'être considérée pour ce qu'elle est: un enjeu de santé publique» (p. 5). Ainsi, depuis la loi du 7 mars 2016 relative au droit des étrangers, elle n'est pas prise en charge par le ministère de la Santé mais par l'Office français de l'immigration et de l'intégration, placé sous l'autorité du ministère de l'Intérieur, témoignant déjà de la grille de lecture privilégiée pour ces questions, ce qui n'est pas sans interroger la manière dont on envisage l'«accueil» des populations réfugiées.

Pour pallier cette situation alarmante, le constat est suivi d'un ensemble de préconisations. Il s'agit tout d'abord d'améliorer la prise en charge matérielle des réfugiés, et tout particulièrement des MNA, afin de leur garantir un accès effectif aux soins. Il est ensuite urgent de mieux former les professionnels de l'accueil sur ces questions (et pas uniquement les spécialistes du soin), ce qui nécessite dans un premier temps de renforcer la connaissance des réalités psychotraumatiques chez les réfugiés, en menant des études épidémiologiques d'envergure nationale. Enfin, le rapport propose des pistes pour restructurer le système de soin: favoriser l'interdisciplinarité et le travail en réseau, systématiser le recours à l'interprétariat professionnel, et adapter les structures de soin existantes aux besoins spécifiques des populations exilées.

Apolline Meyer

\section{Le train d'Erlingen ou La métamorphose de Dieu}

Boualem Sansal, Paris, Gallimard, 2018, 256 pages, 20 euros.

Voici le nouveau Sansal! Comme souvent, la sortie d'un de ses romans ne laisse pas indifférent: 


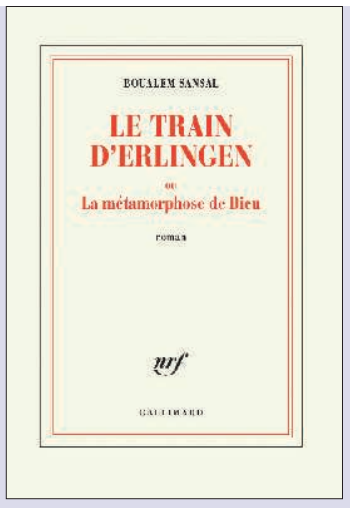

louanges ou détestations déchirent le Landerneau. D'année en année, deux camps se toisent. Les uns éreintent le livre (David Caviglioli dans L'Obs), les autres encensent la vigie de Boumerdès (Félicia-France Doumayrenc sur actuallite.com ou l'excellent Achour Wamara sur exilience.fr). Les uns taisent ce qui dérange, les autres en rajoutent sur ce qui arrange. Sous l'instrumentalisation, la littérature étouffe. De l'écrivain, ne reste que des caricatures. Même s'il ne rechigne pas à faire son office médiatique, il n'est pas certain que l'humble Sansal en demande autant. Aussi, plutôt que de céder à la faiblesse qui veut le critique omniscient, un brin donneur de leçons à défaut de distiller de l'information, il faut ici tenter une expérience. Plutôt que de rendre compte de ce livre, avec une pertinence relative, tentons d'exciter curiosité et réflexion par quelques questions (la liste n'est pas exhaustive) soulevées par ce récit protéiforme, volontairement (sans doute) déstabilisant. Il faut bien que l'écrivain instrumentalisé rende la monnaie de leur pièce aux ventriloques et à ce qu'ils croient être leur marionnette.

Petit résumé pour repère:

Élisabeth Potier, professeur d'histoire dans un lycée de banlieue à la retraite, victime collatérale des attentats de novembre 2015, se réveille (invente) Ute Von Ebert, richissime héritière retirée à Erlingen. Plutôt coincée, car la ville, qui patauge dans «l'essentiel et le superflu», "le défendu et le nuisible», «l'ennui» même et la misère postindustrielle, est sous la menace d'«envahisseurs» invisibles et semeurs de mort. Chacun attend l'arrivée d'un train miracle pour s'esbigner et abandonner la cité. Élisabeth, alias Ute, destine à sa fille des lettres, des notes de lectures et un roman inachevé qui consigne les micmacs de cette ville assiégée et l'histoire de la famille Ebert. Tout est fiction, et la mort d'Élisabeth sonne la fin de la «fantasmagorie». Retour au réel, Erlingen s'efface pour une bonne vieille cité de banlieue et la découverte, par sa fille, des travaux d'écriture maternels. À elle de finir le boulot. La construction du roman déroute, mais la vérité, suggère l'auteur, n'est pas une rectiligne avenue à sens unique (malgré les modes, les faiblesses et les coups de trique), et la langue de Sansal confirme qu'il est et reste un des grands écrivains contemporains, manieur de plume, de mots, d'images hors pair. Et si Sansal se jouait de ses lecteurs par trop idéologiques en les perdant dans les méandres de la fiction et du réel, dans les détours d'une construction baroque où se cache sa part de vérité qu'il veut croire être aussi la nôtre? Alors questionnons, histoire de ne pas réduire la lecture à un règlement de compte à Ok Corral. Ainsi, pourquoi situer ce récit en Allemagne, à Erlingen, imaginaire et paisible ville européenne? Que disent Erlingen et sa société de la modernité, du «vivre ensemble», des édiles et des démocraties où «l'administré» remplace «le peuple»? Pourquoi ce train qui fait paradoxalement penser aux trains de la mort? Pourquoi réincarner Élisabeth en une héritière d'un empire construit par un colon «négrier chez les Indiens» («le seul moteur de l'humanité est le vol et l'escroquerie» disait Cossery)? Que disent de la littérature ces jeux entre fiction et réalité, ces dédoublements, ces allers-retours dans le temps, ces mises en abîme des textes? D'ailleurs, Sansal a-t-il écrit un roman ou cède-t-il à son «obsession» islamiste? Pourquoi alors convoquer Baudelaire et son "que cherchent-ils au Ciel, tous ces aveugles?»? Que viennent faire Kafka, Thoreau, Buzzati ou ce Traité des trois imposteurs, Moïse, Jésus, Mahomet? De quelles métamorphoses est-il question? Et cetera, et cetera.

Mustapha Harzoune

\section{Mille petits riens}

Jodi Picoult. Traduit de l'anglais (États-Unis) par Marie Chabin, Arles, Actes Sud, 2018, 592 pages, 23,50 euros.

\section{JODI PICOULI}

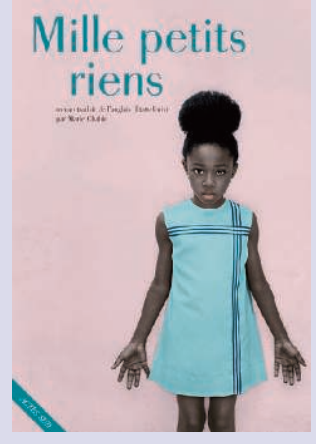

On a beau être une infirmière chevronnée et appréciée, une veuve de guerre courageuse, une mère attentive, s'être éloignée du ghetto pour offrir à son fiston une promesse de vie meilleure, se tenir à l'écart de l'entre-soi; on a beau, depuis les premières classes de l'école, avoir joué le jeu de l'intégration, être «deux fois meilleur», donner des gages, paraître insensible au désarroi collectif..., un jour, «mille petits riens» peuvent vous rattraper, faire écrouler une vie heureuse, bâtie moins sur le mensonge que sur le déni. Car, aux États-Unis, être Noir ne signifie rien. Seul le Blanc serait irréprochable. En un instant, le monde de Ruth va s'écrouler.

Un matin, un comme des milliers d'autres, Ruth prend son service. Elle donne les premiers soins à un bébé né dans la nuit et à sa mère. L'attention du père pour les siens ne parvient pas à masquer l'extrême 
tension qui règne dans la pièce. Et pour cause: Turk et Brittany sont des suprémacistes blancs! Militants radicaux, ils ne supportent pas que cette Noire touche leur fils! Marie, sa supérieure, entérine la volonté du couple. Tout aurait pu en rester là, Ruth aurait pu ravaler sa rancœur, déguiser sa frustration en humour, privilégier son travail, le statut quo et, comme tout un chacun autour d'elle, faire comme s'il ne s'était rien passé. Mais les urgences de l'hôpital conduisent Ruth à devoir surveiller, seule, le nouveau-né. Juste quelques minutes mais, à la clé, la mort du nourrisson. C'est alors que tout bascule. C'est alors que ces milles petits riens, jusque-là invisibles, prennent un sens. Ruth est accusée de meurtre!

Jodi Picoult charpente son récit jusqu'à faire de son lecteur un (heureux) captif, prisonnier de ce cliffhanger aux méthodes dignes des meilleures séries télévisées. Il s'agit bien sûr de racisme, celui lourdingue des suprémacistes blancs, mais surtout celui invisible, sournois, inconscient qui se cache dans ces milles petits riens du quotidien, ces regards, attitudes, mots, réflexes..., en apparence bénins, qui se glissent, structurent, souterrainement, les relations sociales, et parfois amicales. Sans caricatures, trois points de vue rythment le récit: celui de Ruth, celui de Turk et, peut-être le plus important, celui de Kennedy, l'avocate commise d'office, dont le lecteur suit les transformations à mesure que s'approfondit sa relation avec Ruth. En toile de fond, deux univers professionnels et surtout deux fonctionnements institutionnels: celui de la justice (avec le procès et sa préparation) et la santé.

Les quelques bémols possibles (rares répétitions ou ce final qui transporte le lecteur des années après le drame) relèvent du détail: le livre de Jodi Picoult est une réussite et son propos dit, révèle ce qui structure, depuis les institutions jusqu'au tréfonds des mentalités et des imaginaires, la société américaine en matière de relations interraciales.

L'étonnant maintenant: Jodi Picoult, auteure à succès, n'est pas Noire. «Je ne savais pas ce que c'était de grandir dans la peau d'un Noir dans ce pays. Qu'est-ce qui m'autorisait à écrire sur une expérience que je n'ai pas vécue?» Sa réussite tient au fait qu'elle n'écrit pas «à la place de» ou «au nom de». Elle écrit pour montrer comment et quand «les pensées racistes» noyautent les imaginaires, les attitudes, les comportements, l'organisation collective d'une société. Elle écrit dans le droit fil de cette citation de Benjamin Franklin, placée en exergue: «La justice ne pourra être équitablement rendue tant que ceux qui ne sont pas concernés ne s'indignent pas avec ceux qui le sont. » Aux États-Unis, mais rien n'interdit d'élargir le cercle. M. H.

\section{La langue de personne}

Sema Kiliçkaya, Paris, éd. Emmanuelle Colas, 2018, 192 pages, 15 euros.

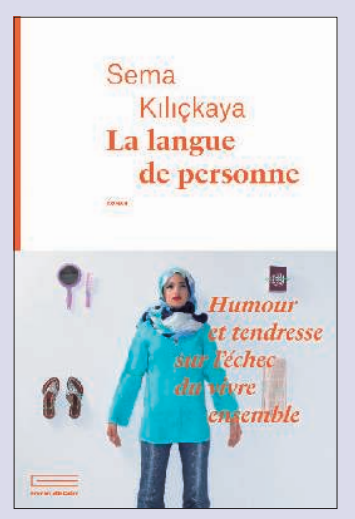

Il y a 25 ans, Fatma imprima une bifurcation dans sa vie, une bifurcation en forme de rupture. Couper le fil d'une narration engageait plus que son existence. En partant, elle rejetait les siens, un amour, un nom, une culture, un pays, une langue. Sauf à se perdre complètement, il y a des retrouvailles auxquelles on ne peut échapper. Fatma, devenue Victoria par la magie de la «naturalisation», revient: son père est mourant et sa mère perd la raison, « le ventre de papa et la tête de maman». Retour dans l'appartement familial et cette HLM où les ombres du passé sont poussées vers le néant par les froids assauts du temps. La mémoire de Fatma "s'affranchit des interdits qu'elle s'était imposés». Elle tourne, telle une "lumière stroboscopique», réveillant de vieux souvenirs, éclairant le présent de couleur sépia.

La généreuse tunisienne du quatrième est partie, avec elle son couscous au «goût du paradis perdu». À sa place: une convertie de 13 ans, déguisée d'une burqa, et la majorité des femmes de la cité qui sont «fermées» (voilées). Elle est loin «la maison de grand-mère» qui sentait «le lait sucré», «l'enfance», «l'indulgence» à l'heure où l'on meurt à Charlie Hebdo. Envolée aussi la cadette. Elle s'est enfuie! Des trois sœurs, il ne reste que l'aînée, Elif, qui a enterré ses rêves sous des couches de silence, de frustration, de sacrifice. De ressentiment. Baba ne veille plus sur la famille, c'est désormais le Pacha qui commande. Lui qui numérote les bananes pour en vérifier la consommation et qui interdit à sa femme de se maquiller. Restent les enfants d'Elif. Décontractés, bruyants, à l'image de Demla qui se la joue «Muslim», cache son ignorance derrière les provocations d'une langue nébuleuse. Pour s'être redressés, «ces jeunes hypertrophiaient ce qu'on leur demandait d'atrophier. Ce faisant - et c'était là leur erreur -, ils mettaient en lumière la part la plus ténébreuse de la culture d'origine». La mère yoyote, cherche sa robe. "Quintessence [...] d'un rêve déchiqueté par la réalité», cette robe est celle qu'elle suspendait pour la protéger de la voracité des rats dans ces taudis réservés aux immigrés!

Un verre de Jurançon à la main, Fatma observe, comme extérieure. Elle se permet de corriger les fautes 
de français de sa nièce, de ramener sa science. Le ton est à l'humour, mais l'attitude est lourdingue. Elle consigne ses réflexions sur un carnet, note les mots des uns et des autres, les transformations et inventions des langues. Leur disparition aussi, «la migration de mes parents m'avait arrachée à ma langue natale». Les langues tournent en boucle, réseau, ramification, circulent, se font et se défont. Comme chez Amin Maalouf, ici «chaque langue vibre à l'écho d'une autre». Pourtant, les vertus du bilinguisme semblent s'arrêter à l'anglais et les cultures « révérées» sont rarement celles «de nos parents». Pire, au tribunal de l'Histoire, «le casier judiciaire» de certains pays reste étonnamment vierge, quand d'autres sont surchargés. Et, à l'heure où «les frères de mes parents tombent comme des mouches», qui dira la trahison de l'idéal européen? Sema Kiliçkaya, Boualem Sansal, Fawaz Hussain, Negar Djavadi...? Une langue de poètes, submergée sous le grand robinet de l'information continue.

Victoria note et morigène. Mais il faudra ôter le masque. Il faudra que Fatma entende le «Tu m'as laissée seule» de sa sœur, qu'elle réponde à la question de Baba, "Pourquoi es-tu partie?». Il faut reprendre le récit. Retrouver cette langue qui, débarrassée des illusions de l'origine ou de son déni, fasse de chacun des personnes et restaure ce qu'Arendt appelle dans son Journal de Pensée «l'équivocité chancelante du monde».

Sema Kiliçkaya ne donne pas dans la caricature télévisuelle et cinématographique de la Musulmane opprimée. Sesindignations vont au-delà. Elle montre les résistances, la complexité des êtres, des trajectoires. Les raisons d'espérer. Sa force de conviction est essentielle, réconfortante. La langue de personne a reçu le Prix France-Turquie 2018.

M. H.

\section{Huit ans au pouvoir. Une tragédie américaine}

Ta-Nehisi Coates. Traduit de l'anglais (États-Unis) par Diana Hochraich, Paris, Présence africaine, 2018, 308 pages, 24,90 euros.

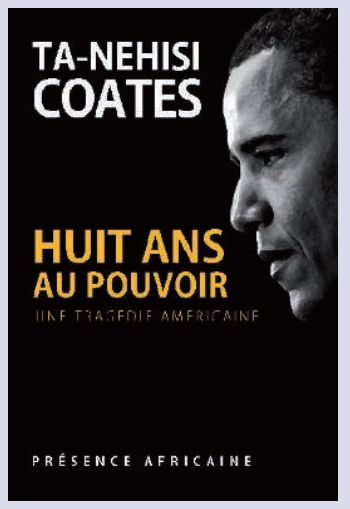

Quel livre! Ou plutôt quels articles! Car Coates est journaliste et cette publication rassemble huit papiers écrits tout au long de la présidence Obama pour le site d'information The Atlantic. Huit papiers remis chacun en perspective par une introduction rédigée ex post, où l'auteur donne les conditions de leur écriture, les discute, les commente, les critique même. Si l'auteur se raconte, ce n'est pas par nombrilisme. Lorsque Coates revient sur son enfance, sur sa famille, sa mère enseignante, son père membre des Black Panthers, ses années d'école, son mariage, ses doutes et ses réussites, le hip-hop, sa vie d'Afro-Américain né en 1975 du côté de Baltimore, ou lorsqu'il évoque la mort de son ami Prince Jones abattu en 2000 par un policier..., c'est pour dire et montrer les soubassements, les ressorts de sa pensée, ses grilles de lecture. Ce n'est pas pour faire le kéké que Coates parle de lui, mais, semble-til, par honnêteté intellectuelle. L'infiniment petit n'est pas repli narcissique mais volonté de traduire l'infiniment grand. Ou quand l'expérience individuelle rejoint l'expérience collective.
Ce qui frappe à la lecture de ces longs papiers, c'est le talent de l'auteur: brio des reportages (Chicago, Obamacare, incarcération des Noirs...) et des portraits (Michelle ou Barack Obama, Bill Cosby ou des anonymes); hauteur de vue des réflexions; densité des raisonnements structurés autour de récits d'expérience et de témoignages, de références et de sources multiples, universitaires, statistiques, et surtout historiques. Car le tragique ici s'enracine dans l'histoire du pays, dans le pillage des Noirs, depuis l'esclavage jusqu'aux subprimes, en passant par l'exploitation salariale, les lois Jim Crow, les détentions massives. Pour Coates, l'existence et la prospérité des États-Unis ont pour fondement la violence faite aux Noirs. Au pays dit de la liberté, "c'est l'esclavage qui a permis la démocratie».

L'élection d'Obama, ses discussions avec le président ont pu faire vaciller le pessimisme et la «défiance» de Ta-Nehisi Coates. L'intelligence du président, sa confiance en l'Amérique auraient pu emporter les doutes. C'était compter sans la rigueur des analyses du journaliste. Sans son expérience. West Baltimore n'est pas Hawaï. Avoir des grands-parents blancs aimants n'a rien de commun avec une enfance passée dans « un monde à part», où jamais il ne croisa de Blancs. Obama et Coates, ce sont deux regards différents, deux histoires américaines.

National Book Award pour Between the World and Me (Une colère noire, Autrement, 2016), Ta-Nehisi Coates appartient à cette génération d'intellectuels afroaméricains plus proches de Baldwin ou de Malcom $X$ que de Martin Luther King. Pour lui, la question raciale, entendre la suprématie blanche, est et reste, même après la victoire d'Obama, au cœur de l'histoire américaine. Surtout après: l'élection de Trump en est la parfaite illustration.

En 1835, Gustave de Beaumont écrivait, dans Marie ou l'esclavage 


\section{Les travailleurs chinois recrutés par la France pendant la Grande Guerre}

Yves Tsao, Aix-en-Provence, Presses universitaires de Provence, 2018, 366 pages, $25 €$.

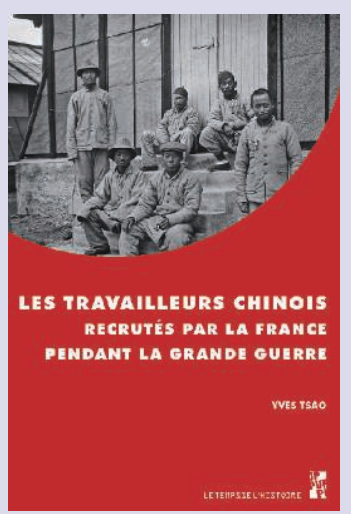

Avec cet ouvrage, fruit de son travail dans le cadre de l'École des hautes études en sciences sociales, Yves Tsao contribue à faire sortir de l'oubli un épisode important de la Première Guerre mondiale: la venue en France, à partir d'août 1916, de 37000 travailleurs chinois. S'inscrivant ainsi dans l'enjeu mémoriel posé par la commémoration du centenaire de laGrande Guerre, Yves Tsao s'en détache par la suite, en se plaçant résolument du côté de l'histoire.

La précision des notes de bas de page, le recours à des tableaux et graphismes, l'approche tout à la fois chronologique et thématique:

Yves Tsao veut faire parler les faits, dans un souci d'objectivité et d'exhaustivité, et s'attache

à retracer le parcours de ces 37000 travailleurs chinois dans sa totalité. L'étude s'ouvre ainsi sur une description détaillée des opérations de recrutement, nées de la rencontre entre le besoin de main-d'œuvre de l'administration française de la Guerre et la volonté des autorités chinoises de se rapprocher des Alliés, afin de se protéger des visées japonaises. Elle s'achève par le détail du processus de rapatriement mis en place dès l'armistice, et l'évocation des modalités qui permirent à quelques centaines de Chinois de s'installer durablement en France, participant ainsi «à la constitution de la première communauté chinoise».

Les parties intermédiaires de l'ouvrage s'intéressent aux conditions d'existence et à l'environnement dans lequel évoluent les travailleurs chinois. En proie aux pénuries et à l'isolement, ils doivent faire face à l'hostilité de populations locales dont les préjugés sont façonnés par le discours colonial de l'époque, ainsi que par une littérature à sensation qui véhicule des représentations racistes et xénophobes. Yves Tsao met l'accent sur les éléments les plus originaux qui émergent de ses recherches, en insistant notamment sur les formes de résistance déployées par les travailleurs chinois face au non-respect des contrats d'engagement et aux violences subies: revendications multiples, grèves et organisation en association, autant de tentatives infructueuses pour tenter d'améliorer leurs statut et conditions de vie. L'autre intérêt majeur de cette étude réside dans l'éclairage qu'elle pose sur les réactions du syndicalisme, face à un monde ouvrier qui voit dans ces travailleurs «des concurrents visant leurs emplois ou les responsables du maintien au front de leurs camarades».

Monographie très détaillée et complète, cette étude s'inscrit dans le propos plus large du renouveau historiographique qui, depuis la fin des années 2000, s'intéresse à la manière dont la Grande Guerre ouvre «durablement des routes migratoires empruntées, en fonction de circonstances particulières et selon des temporalités propres, par des vagues successives d'émigrants originaires de Chine, du Vietnam et du Maghreb», constituant ainsi un moment charnière de l'histoire de l'immigration. Yves Tsao prend ainsi la suite des travaux de Laurent Dornel qui, avant de publier Les étrangers dans la Grande Guerre en 2014, avait présenté, sur le site du Musée national de l'histoire de l'immigration, un dossier sur les travailleurs étrangers, coloniaux et chinois pendant la Première Guerre mondiale. Le présent ouvrage, de par sa précision et la richesse de son contenu, contribue à éclairer un peu plus cette histoire.

On peut cependant regretter, en raison même de cette qualité et de l'exigence du propos, le fait que l'auteur n'ait pu consulter de sources chinoises, principalement en raison de la barrière de la langue. En 2014, une conférence, organisé par l'Institut français de Pékin, a réuni autour de cette question des historiens français comme chinois: espérons que cette initiative va permettre de continuer à croiser les regards sur un épisode essentiel de ces deux histoires nationales.

Yasmine Achouche 